\section{Introduction}

For pharmaceutical products with several active compounds, high performance liquid chromatography (HPLC) is a recognized method for multicomponent analysis. The strength of HPLC is the resolution of multiple components. However, HPLC requires specialized instrumentation, and isocratic analysis of each sample can take a minimum of 5-10 minutes.

Although UV-visible spectroscopy is widely used in pharmaceutical applications, it has been used limitedly for multicomponent analysis of pharmaceutical products. Multicomponent analysis using multiple discrete wavelengths or a continuous spectrum has been successfully applied when spectral overlap among components was minimal and the contribution by each component to the absorbance of the mixture was independent of the other components. In pharmaceutical applications, multicomponent analysis was done by multiple linear regression with instrument based computers for determination of two, three and four component mixtures ${ }^{1-3}$.

\section{Features of FSQ}

FSQ derives the Fourier transform of the absorbance spectrum. The Fourier transformation results in data reduction, 70 terms per spectrum, with optimal information retention over the wavelength range, and the signal-to-noise ratio is increased. The lower Fourier terms contain information of the background absorbance in the spectrum and the higher terms contain high frequency noise information. FSQ allows the elimination of these Fourier terms in the analysis. The standards used to establish a calibration matrix in FSQ are mixtures of the components to be analyzed. Principal component analysis is applied to the Fourier transform of the standard mixtures, and a set of orthogonal eigenvectors and eigenvalues are derived. The number of significant eigenvalues represents the spectrally distinct chemical components in the mixtures. When the number of eigenvalues is larger than the number of components, this suggests that there are matrix effects, impurities and/or interactions between components. In this situation, the additional eigenvectors account for these effects and

\title{
Full Spectrum \\ Quantitation: An Advanced UV/Visible Spectroscopic Method For Multicomponent Dissolution Testing
}

\author{
Michael H. Simonian, Ph.D. \\ Senior Staff Scientist, Beckman Instruments, Inc. \\ Analytical Development Center, Fullerton, Calif.
}

Full Spectrum Quantitation (FSQ) is an advanced spectroscopic method for multicomponent analysis that is based on principal component regression and uses Fourier transform preprocessing of the absorbance spectrum ${ }^{4}$. FSQ has been successfully applied to multicomponent dissolution testing of two different pharmaceutical formulations: one contained pseudoephedrine hydrochloride and chlorpheniramine maleate; the other contained phenylpropanolamine hydrochloride and chlorpheniramine maleate ${ }^{5}$.

This article will describe the features of FSQ and outline the steps to perform an analysis. Also, the analysis of a three component pharmaceutical formulation by FSQ will be compared to that by HPLC. can be retained for the calibration. Regression analysis is used to correlate the orthogonal representations to the concentrations of the components in the standard mixtures. The derived regression coefficients are used to quantitate the components in an unknown sample from its Fourier transformed spectrum.

FSQ addresses the limitations of previous spectroscopic methods of multicomponent analysis.

- Vector analysis of the spectrum allows components with considerable overlap to be analyzed.

- FSQ accounts for interactions between components, which could result in deviations from the BeerLambert law, and these interactions are included in the calibration. (continued on page 4) 
(continued from page 3)

- If the unknown contains a component(s) that contributes a background absorbance but does not require quantitation, this component can be left out of the calibration set. The effects of these components, for example excipients, are eliminated by not using the lower Fourier terms for the calibration ${ }^{6}$. mixtures and including a baseline of $10-20 \%$ of the spectrum. The last three steps in the above protocol are done with FSQ.

The validation of the FSQ calibration is done with controls that are mixtures of components at concentrations other than those used for the calibration standards. The validation is confirmed by comparison

\section{Full Spectrum Quantitation: An Advanced UV/Visible Spectroscopic Method For Multicomponent Dissolution Testing}

of the standards' error term, the standard error of estimate (SEE), to the controls' error term, the standard error of prediction (SEP). The standards need not be prepared and read before each analysis of unknown samples. Instead, a previously saved standard file can be recalled and several freshly prepared controls

\section{Steps To Perform FSQ}

The protocol for FSQ involves five steps: ?

1. Determine the concentration range of each component that has a linear absorption relationship.

2. Prepare standards, which are mixtures of components, and scan selected mixtures to determine the analytical wavelength range.

3. Perform calibration step with the standard mixtures.

4. Validate the calibration with control mixtures.

5. Analyze unknown samples.

The first three steps are performed once initially, and subsequent analyses only require the last two steps.

To determine the concentration range that is linear to absorbance, single component analysis is done for each component. The concentration range of each component in the standard mixtures must bracket the expected component concentrations in the unknown. Prepare a number of standard mixtures that is at least equal to the number of components. Overspecifying the number of standards will increase the accuracy of the calibration regression coefficients. The analytical wavelength range used in FSQ is ascertained by scanning selected standard can be read prior to an analysis. If the SEP for these controls is similar to that of the original SEE, then the analysis of samples can be performed using the recalled standard calibration set. If these error parameters do not agree, then new calibration standards should be prepared and read prior to analysis of the samples.

\section{Comparison of FSQ to HPLC}

The capability of FSQ will be evaluated and compared to HPLC in the quantitation of a mixture of components found in an over-the-counter analgesic ${ }^{8}$. The components are acetaminophen, aspirin and caffeine.

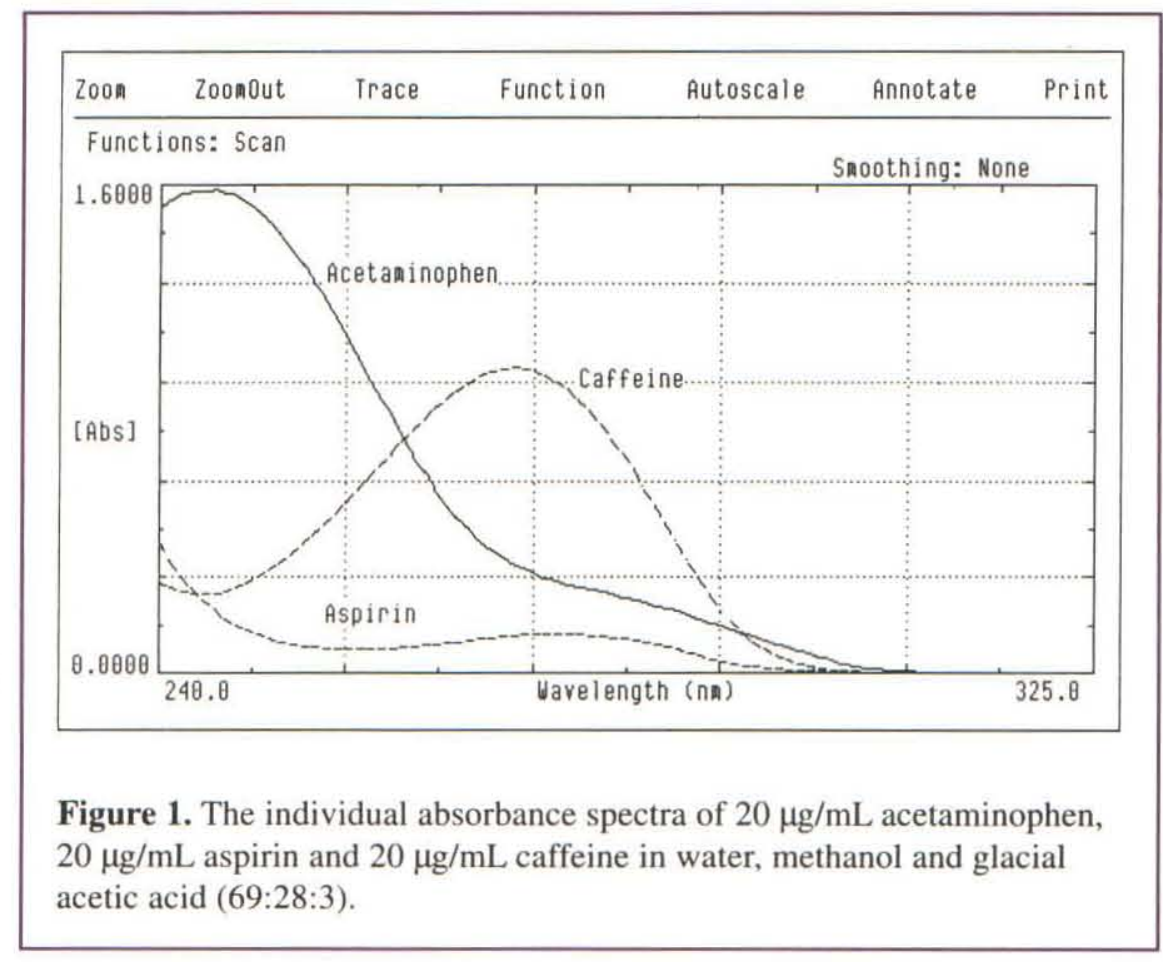




\section{Materials and Methods}

The sample tablets with a label claim of $250 \mathrm{mg}$ acetaminophen, $250 \mathrm{mg}$ aspirin and $65 \mathrm{mg}$ caffeine were Excedrin Extra Strength Pain Reliever (BristolMyers Products).

HPLC analysis was performed on a Beckman Instruments System Gold equipped with the programmable 502 Autosampler, 126 solvent delivery module with programmable eight solvent selection, the 166 variable UV detector and controlled with Gold Software. Reversed-phase chromatography was done with an Ultrasphere C-18 column $(4.6 \mathrm{~mm} \times 150 \mathrm{~mm}, 5 \mu$ particle size) at $23^{\circ} \mathrm{C}$ with an isocratic mobile phase at a flow rate of $2 \mathrm{ml} / \mathrm{min}$. The mobile phase composition was water, methanol and glacial acetic acid (69:28:3). Components were detected by absorbance at $280 \mathrm{~nm}$. For each component, standards were prepared in the mobile phase and a calibration was done by a least squares fit. The concentration ranges of the standards for each component were $65-85 \mu \mathrm{g} / \mathrm{ml}$ for acetaminophen and aspirin, $12-28 \mu \mathrm{g} / \mathrm{ml}$ for caffeine, and $0.5-16.5 \mu \mathrm{g} / \mathrm{ml}$ for salicylic acid.

Multicomponent analysis by UV-visible spectroscopy was done with a Beckman Instruments DU 650 spectrophotometer equipped with FSQ.

Spectra were measured with a $10 \mathrm{~mm}$ quartz cell. All standard mixtures, validation mixtures and samples were prepared in water, methanol and glacial acetic acid $(69: 28: 3)$ The analytical wavelength range for FSQ was $240-325$ $\mathrm{nm}$. Fifteen standard mixtures were used for calibration and the concentrations of acetaminophen and aspirin were $10,12.5$ or $15 \mu \mathrm{g} / \mathrm{ml}$, and those for caffeine were 2 ,
3 or $4 \mu \mathrm{g} / \mathrm{ml}$. These concentrations correspond to 80 , 100 or $120 \%$, respectively, of the label claim after a 200 -fold dilution of a single tablet dissolved in $100 \mathrm{ml}$ of solvent. The calibration standard mixtures were validated with a set of five mixtures made up at component ratios that were different from those used in the respective calibration standards.

Multicomponent analysis by HPLC and FSQ was performed on the same 10 tablet samples from a single batch. Each tablet sample was pulverized, taken up in $100 \mathrm{ml}$ of water, methanol and glacial acetic acid (69:28:3) and sonicated for nine minutes. For HPLC, the samples were diluted 33.33-fold prior to injection. The same samples were diluted 200 -fold and analyzed spectrophotometrically with FSQ. Statistical comparison between the two analytical methods of the results for each component was done by a paired $\mathrm{t}$ test.

\section{Results}

HPLC separation of the three components was achieved within 4 minutes. There was baseline resolution between each component. The retention times for each component were $1.02 \mathrm{~min}$ for acetaminophen, $1.53 \mathrm{~min}$ for caffeine and $3.08 \mathrm{~min}$ for aspirin.

The UV absorbance spectrum of each of the four compounds is shown in Figure 1. The spectra for aspirin and caffeine have considerable overlap. The absorbance spectra of one of the standard mixtures is shown Figure 2. This mixture was used in the calibration for FSQ. None of the distinctive spectral (continued on page 6) 
(continued from page 5 ) elements observed in the scans of the individual components are present in the scan of the mixtures.

The results of the content uniformity determination by HPLC and FSQ are given in Table I. The individual component content for each of 10 tablets are compared between the two analytical methods. Paired comparison of each sample for each component by HPLC and FSQ indicated the results are not statistically different between the two methods.

The results demonstrated that the advanced spectrophotometric method, FSQ, gave equivalent results to those by an accepted and approved HPLC method for multicomponent analysis of a dosage formulation with acetaminophen, aspirin and caffeine. It should be emphasized that accurate quantitation of these components by FSQ was achieved even with significant spectral overlap of two of the components, aspirin and caffeine (Figure 1).

\section{Conclusion}

The spectrophotometric method FSQ proved to be suitable for analysis of a multicomponent pharmaceutical product with three active ingredients. FSQ has been verified as accurate as an approved HPLC method of analysis for this multicomponent formulation.

The advantage of FSQ is the speed of sample analysis as compared to HPLC. In this study, the HPLC separation and analysis of each sample required $4 \mathrm{~min}$. In contrast, these mixture were read and analyzed by FSQ in less than $10 \mathrm{sec}$ per sample. FSQ is self contained and built into the spectrophotometer and therefore requires no off-line computations or user pre-processing of the spectral data. FSQ uses standards that are mixtures of components and thus takes into account any chemical interaction and subsequent effects on the spectra. These characteristics of FSQ make it a useful alternative to HPLC for pharmaceutical multicomponent analysis, including dissolution testing ${ }^{5}$.

\section{TABLE I}

Results By HPLC And FSa

Concentration (mg/tablet)

\begin{tabular}{lllll} 
FSQ & \multicolumn{2}{c}{ Aspirin } & \multicolumn{2}{c}{ Caffeine } \\
224.3 & HPLC & FSQ & HPLC & FSQ \\
240.4 & 243.5 & 225.2 & 66.1 & 59.6 \\
246.3 & 244.3 & 240.3 & 69.1 & 67.0 \\
239.4 & 247.8 & 235.9 & 69.0 & 66.5 \\
253.1 & 247.3 & 235.9 & 67.5 & 65.3 \\
261.7 & 241.3 & 243.1 & 65.8 & 68.3 \\
229.4 & 238.3 & 234.4 & 64.2 & 65.1 \\
255.5 & 235.7 & 248.0 & 66.6 & 67.8 \\
235.5 & 234.0 & 246.3 & 67.1 & 67.7 \\
249.7 & 240.4 & 228.4 & 66.3 & 63.2 \\
*P>0.40 & 242.6 & 238.3 & 65.7 & 66.0 \\
\hline \multicolumn{5}{c}{ *P $>0.20$} \\
\end{tabular}

tween HPLC and FSQ analysis of each component.

Table reproduced by permission from Spectroscopy.

\section{References}

1. J. M. Hoover, R.A. Soltero, and P.C. Bansal, J., Pharm. Sci., 76, 242-244 (1987).

2. J.L. Murtha, T.N. Julian, and G.W. Radegaugh, J., Pharm. Sci., 77, 715-718 (1988).

3. G. Sala, S. Maspoch, H. Iturriaga, M. Blance, and V. Cerda, J., Pharm. Biomed. Anal. 6, 765-772 (1988).

4. M.H. Simonian and C.W. Brown, Beckman Technical Information Bulletin T-1795A, pp. 1-8, (1995).

5. S-C. Lo, S.M. Donahue, and C.W. Brown, J., Pharm. Sci., 82, 350-354 (1993).

6. Simonian, M.H., S. Dinh and T. Li, Pittsburgh Conference, Abstract 632, (1995).

7. Simonian, M.H., Beckman Technical Information Bulletin T-1717B, pp. 1-7, (1994).

8. Simonian, M.H., S. Dinh, LA. Fay, Spectros copy, 8, 37-42, (1993). 